\title{
Meeting report on Synthetic Biology Young Scholar Forum
}

\author{
Zhen $\mathrm{Xie}^{1,3, *}$ and Junbiao $\mathrm{Dai}^{2,3, *}$ \\ ${ }^{1}$ Bioinformatics Division, TNLIST/Department of Automation, Tsinghua University, Beijing 100084, China \\ 2 School of Life Sciences, Tsinghua University, Beijing 100084, China \\ ${ }^{3}$ Center for Synthetic and Systems Biology, Tsinghua University, Beijing 100084, China \\ * Correspondence: zhenxie@tsinghua.edu.cn, jbdai@tsinghua.edu.cn
}

Received October 10, 2015

On May 23 in 2015, the first Synthetic Biology Young Scholar Forum (SynBioYSF) was held at Tsinghua University in Beijing, China. This forum aimed to serve as a platform for synthetic biologists, especially early career scholars, to exchange and discuss recent advances and promote synthetic biology research in China. Drs. Zhen Xie and Junbiao Dai served as the secretary general and deputy secretary general in the first SynBioYSF organization committee, which included eleven young principle investigators in the field of synthetic biology. This forum attracted about 200 participants from more than 40 universities, institutes and enterprises. In this report, we summarized 19 invited presentations and 4 lightening talks in this forum.

\section{KEYNOTE TALKS}

The first keynote speech was given by Dr. Guo-Ping Zhao (Shanghai Institute for Biological Sciences, Chinese Academy of Sciences, China) focused on synthetic biology for applied biotechnology. Dr. Zhao began his talk by looking back to 1950-1980's when they were focused on the development of bacterial fermentation technology with forward genetics-based gene mapping and biochemistry-based functional analysis of fermentation related enzymes. With the introduction and development of powerful tools of molecular biology and genomics since 1990's, bacterial physiology has been studied based on a broader spectrum of complete nature blueprints employing recombinant technology in industrial strains, both in vitro and in vivo, for better and broader applications. Thus, microbial physiology was transformed to systems biology based on omics analyses, which facilitated the quantitative understanding of the nature of biological processes and the improvement of production of biological molecules via microbial metabolic and protein engineering. Since the beginning of this century, bottom-up forward engineering strategy and robust "standardized" technology platforms have been explored via sophisticated innovation geared by the emerging area of research that enables both the design and construction of novel artificial biological pathways, organisms or devices, or the redesign of existing natural biological systems. At last, Dr. Zhao pointed out that although synthetic biology is a long march for the rebirth of life science and biotechnology in the fire of convergence innovation, by integrating well-coordinated dedicated research teams, it is going to grow up and to revolutionize our understanding of life and our capability of industrialization of biology for the wealth and health of human beings.

The second keynote speaker is Dr. Qi Ouyang (Peking University, China). Dr. Ouyang first gave an overview on the development in synthetic biology in the past decade, which has raised the attention of the research organizations all over the world. He then discussed the current stage of synthetic biology and the main tasks in the field. $\mathrm{He}$ introduced one work in which they programmed a genetic circuit that executes Pavlovian-like conditioning, an archetypical sequential-logic function, in Escherichia coli [1]. He emphasize that at present application of synthetic biology to biological engineering is in the early stage, waiting for major breakthroughs in synthetic biology; and major breakthroughs will certainly appear in the boundaries of biology, mathematics, physics, chemistry, computer science, and engineering. In this 
sense, he thinks that synthetic biology is an interdisciplinary science, rather than simply biological engineering.

The last keynote speech was presented by Dr. Peng Yin (Harvard University, USA), in which Dr. Yin discussed how to use DNA to construct and visualize nanoscale structures. He first gave an overview of his lab's work in DNA nanotechnology. They have invented a general framework to program DNA/RNA strands to selfassemble into structures with user-specified geometry or dynamics. By interfacing these nanostructures with other functional molecules, they have introduced digital programmability into diverse application areas, e.g., fabrication of inorganic nanoparticles with arbitrary prescribed shapes, robust DNA/RNA probes with near optimal binding specificity, and RNA-based translation regulators with unprecedented dynamic range and orthogonality $[2,3]$. Next, he described their recent work on DNA-based super-resolution imaging. Due to limitations in current optical microscopy, scientists face three challenges when attempting to image biology on the molecular scale: (i) blurred vision (i.e., difficulty to clearly visualize individual molecules for crowded targets), (ii) (partial) color blindness (i.e., difficulty to simultaneously track distinct species with many colors due to spectral overlap), and (iii) ambiguous quantification (i.e., difficulty to precisely count the number of targets in a resolution-limited area). Using programmable fluorescent DNA probes, Dr. Yin's lab developed a highly multiplexed $(10 \times$ demonstrated), precisely quantitative ( $>90 \%$ precision), and ultra-high resolution (sub-5 nm) optical imaging method that promises to simultaneously address these challenges and broadly transform biomedical research $[4,5]$.

\section{ENZYME AND METABOLIC ENGINEERING}

One of the key areas in synthetic biology is to engineer the biological system for production of chemicals or commodities. In this section, the first talk was given by Dr. Hao Song (Tianjin University, China), discussing their recent work on engineering bacterial extracellular electron transfer (EET) pathway for applications in energy and environments [6,7]. Bacterial EET that dictates the exchange of electrons between bacteria and conductive surfaces of electrodes is a major bottleneck in determining the efficiency of microbial electrochemical systems (MES). Based on different mechanisms of EET (i.e., direct-contact via c-type cytochromes, and shuttlemediated EET), they engineered a few well-established exoelectrogenic bacteria by synthetic biology approaches: (i) cell-cell communications (quorum sensing mechanism) to enhance biosynthesis of electron shuttles in Pseudomonas; (ii) exogenous shuttle biosynthesis pathways in Shewanella; (iii) electrochemically active biofilms, as highly structured microbial communities attached on electrodes; (iv) microbial metabolism for electrode respiration; and lastly (v) electrodes' nanostructures in facilitating cell-electrode interactions.

In the second talk of this section, Dr. Yihua Chen (Institute of Microbiology, Chinese Academy of Sciences, China) presented their approaches to delineate the biosynthetic pathway of the carbamoylated D-gulosamine (D-GulN) moiety, a rare sugar presenting in the antimicrobial molecules streptothricins (STNs) [8]. They found an N-acetyl-D-galactosamine that is first attached to the streptolidine lactam by the glycosyltransferse StnG and then epimerized to N-acetyl-D-gulosamine by the putative epimerase StnJ. After carbamoylation by the carbamoyltransferase StnQ, N-acetyl-D-GulN is deacetylated by StnI to furnish the carbamoylated D-GulN moiety. They identified StnG as an unprecedented GT-A fold N-glycosyltransferase that glycosylates the imine nitrogen of guanidine, and StnI as the first reported Nacetyl-D-GulN deacetylase.

Next, Dr. Jin Miao from Institute of Microbiology, Chinese Academy of Sciences, focused on the design and synthesis of biological chassis, parts, device and modules in Streptomyces avermitilis to produce Avermectin B1a. They did a computational study on the de novo biosynthesis of Avermectin B1a, followed by multiplestrategy metabolic engineering to boost the productivity of this compound. The last talk of this section was given by Dr. Jiangyun Wang (Institute of Biophysics, Chinese Academy of Sciences, China). Dr. Wang presented their recent progress on a biosynthetic route for metalloenzyme design [9]. Through the genetic incorporation of the TyrHis ligand and $\mathrm{CuB}$ site into myoglobin, they recapitulated important features of $\mathrm{HCO}$ into the small soluble protein, which exhibits selective $\mathrm{O}_{2}$ reduction activity while generating less than $6 \%$ ROS, at more than 1,000 turnovers. These results support that Tyr-His crosslink is indeed important for HCO function, and creates the exciting opportunity to rapidly evolve better $\mathrm{HCO}$ model proteins to achieve higher activity and selectivity, which may be suitable as alternatives to precious metal catalyst in fuel cells.

\section{CIRCUIT AND GENOME EDITING}

One of main tasks in synthetic biology is to predictably assemble genetic circuits that are composed of DNA parts encoding diverse functions. However, these DNA parts are often functionally interfered with each other and with their host cells. In the first talk of this section, Dr. Chunbo Lou (Institute of Microbiology, Chinese Academy of Sciences, China) described a modular architecture to insulate the operators from their cognate promoters recognized by T7 RNAP or sigma ECF11 in E. coli. Dr. 
Lou developed a simple mathematical model to accurately describe more than 1,000 transcriptional regulatory parts consisted of insulated promoters and operators. Furthermore, the high-performance IFFL circuits were in-silico designed based on the 1,000 regulatory parts, and then experimentally confirmed their predictability. These results suggested that this library of versatile modular parts is a powerful toolbox to facilitate the construction of sophisticated genetic circuits.

Quantitative manners of signaling dynamics such as constant, pulse or oscillatory dynamics encode biological information and often determine cell-fate. Although many individual components of signaling modules have been characterized, it is still challenging to fully understand the relationship of network topology and signal behavior due to the complexity of the signaling networks. Synthetic biology provides a forward engineering approach to study cell behavior in a quantitative and predictive manner by introducing synthetic signaling modules. In the second talk of this section, Dr. Ping Wei (Peking University, China) presented the progress on reconstituting the wellstudied human NF- $\mathrm{KB}$ signaling pathway in yeast cells in which the entire NF- $\kappa \mathrm{B}$ components are missing during evolution. Dr. Wei showed that adjusting the I- $\kappa B$ degradation rate, negative feedback activity and NF- $\mathrm{BB}$ protein concentration can fine-tune the oscillation behavior of synthetic NF- $\kappa B$ circuit. These results demonstrated how synthetic gene circuit could be a powerful tool to uncover the fundamental biology system design principles.

Recent development of the clustered regularly interspaced short palindromic repeats (CRISPR) and CRISPRassociated protein (Cas) system provides an unprecedented capability for precise genome editing and programmable gene regulation. Dr. Lei Stanley Qi (Stanford University, USA) presented his recent progress on the method to repurpose CRISPR/Cas system for sequence-specific gene regulation. Dr. Qi demonstrated that the CRISPR/Cas tools allowed multiplex, inducible activation or repression of gene expression in a variety of organisms, which enabled genome-wide perturbation for studying gene networks $[10,11]$. Dr. Qi showed that the higher-order circuits constructed with the engineered CRISPR/Cas system can be used for modulating metabolic pathways in yeast and controlling motility in mammalian cells [12]. In the last talk of this section, Dr. Wensheng Wei (Peking University, China) reported the development and optimization of the genome-scale CRISPR/Cas knockout library for the identification of host components important for Clostridium difficile and Hepatitis $\mathrm{C}$ infections through function-based screening and high-throughput sequencing analysis $[13,14]$. This genetic screening strategy is a powerful genetic screening strategy, which will have broad application in the discovery of disease mechanism and the identification of new drug targets.

\section{MAMMALIAN CELL ENGINEERING}

In mammalian cells, the complex interaction network of miRNAs and their target RNAs has been shown to allow indirect cross-regulation between different competing endogenous RNAs (ceRNAs) by sequestering shared miRNAs. However, natural ceRNA networks are complex and hard to perturb, which makes it difficult to quantitatively understand the mechanism of miRNAmediated ceRNA regulation. To obtain a comprehensive and quantitative understanding of miRNA regulation principles on competing RNAs, Dr. Xiaowo Wang (Tsinghua University, China) formulated a coarse-grained mathematic model for a minimum miRNA-ceRNA system and engineered a corresponding genetic circuit in cultured human HEK293 cells [15]. Dr. Wang demonstrated that the range and strength of ceRNA regulation are largely determined by the relative abundance and the binding strength of miRNA and ceRNA. Furthermore, Dr. Wang found that a nonreciprocal competing effect between partially and perfectly complementary targets is mainly due to different miRNA loss rates in these two types of regulations, and proposed a design strategy to reduce the siRNA off-target effect in mammalian cells.

The development of systems biology provides a systems-level approach to elucidate complex biological functions, which enables the design and implementation of new biological function via the synthetic biology approach. Dr. Hsueh-Fen Juan (Taiwan University) demonstrated their recent research on understanding of various cancers by using an integrative approach, which has implications for identification of cancer biomarkers and development of potential therapeutic strategies [1619]. In addition, Dr. Haifeng Ye (East China Normal University, China) presented a synthetic insulin-sensitive transcription controller that self-sufficiently distinguished between physiological and pathological blood insulin levels in different mouse models [20]. Dr. Ye showed that the circuit reversed the insulin resistance syndrome by controlling the expression of adiponectin or GLP-1 to attenuate hyperglycaemia and dyslipidaemia, respectively. These results suggested new opportunities for future gene- and cell-based treatments of multifactorial metabolic disorders by engineering synthetic gene network devices to sense pathologic markers and coordinate the expression of therapeutic transgenes.

In the last talk of this section, Dr. Qiong Wu (Tsinghua University, China) presented a universal gene expression enhancement tool, called RNAe [21]. RNAe was derived from a class of long non-coding RNA that functioned at 
the post-transcriptional level. Dr. Wu showed that the 300-nt minRNAe was sufficient and necessary for RNAe function. This region contained a 72-nt $5^{\prime}$ pairing sequence which determines the specificity, a 167-nt short non-pairing interspersed nuclear element (SINE) B2 sequence which enhances ribosome recruitment to the target mRNA, and poly(A) tail. Dr. Wu stated that this novel technology for enhancing the production of proteins would find wide applications in both research and biopharmaceutical production.

\section{ENABLING TECHNOLOGY}

In the first talk of this section, Dr. Yulong Li (Peking University, China) showed their recent progress on engineering two complimentary fluorescent imaging methods for sensing the release of neurotransmitters by hijacking the prototype beta- 2 adrenergic receptor (a GPCR), which could be powerful tool for understanding how neurons transfer information with neural circuits. One method was based on the coupling of GPCR activation and its subsequent endocytosis, while the other one was to convert the ligand-dependent structural rearrangement of GPCRs to the folding of a fluorescent protein. Dr. Li demonstrated that the engineered ultrafast beta-2 sensors displayed a signal to noise ratio exceeding $30 \%$. In addition, using principle of synthetic biology, Dr. Li expanded the sensors to other important molecules, such as adenosine, acetylcholine and neuropeptide. Dr. Li anticipated that the further optimization of the new generation sensors would provide critical spatial and temporal dimension to help deepen our understanding the regulation of neurotransmitter release in health and disease.

In the second talk of this section, Dr. Yizhi Cai (University of Edinburgh, UK) presented the progress on the Synthetic Yeast genome project $(\mathrm{Sc} 2.0$, www. syntheticyeast.org) that aims to design, construct, and replace the native $12 \mathrm{Mb}$ genome of Saccharomyces cerevisiae with a fully synthetic version. Dr. Cai reported that the Sc2.0 international partners include Imperial College London, Edinburgh University (UK), Tsinghua University, Tianjin University, BGI (China) and New York University (USA). Dr. Cai introduced the designer changes of Sc2.0 chromosomes, including removal of genomic destabilizing elements, insertion of synonymously recoded sequences called PCR tags that permit encryption and tracking of the synthetic DNA, and embedding an inducible evolution system called SCRaMbLE (Synthetic Chromosome Rearrangement and Modification by LoxP-mediated Evolution) that can generate combinatorial genetic diversity on command. Dr. Cai suggested that the infrastructure developed to facilitate Sc2.0 genome design and construction is applicable to new projects ranging from single gene/pathway design to synthesizing artificial chromosomes, which has the potential to revolutionize the future of genome structure-function analysis. Dr. Cai also discussed their recent work on Yeastfab, in which they designed and constructed a set of standardized biological parts for metabolic engineering applications [22].

Recently, synthetic biology provided a new route to "mint" novel biomaterials by assembling biomolecules into designed nanostructures. By using a genetic modular design strategy, Dr. Chao Zhong (Shanghai Tech University, China) successfully engineered biomimetic amyloid-based adhesives, in which mussel foot proteins (Mfps) of Mytilus galloprovincialis was rationally fused with CsgA proteins, the major subunit of E. coli amyloid curli fibres [23]. Dr. Chao showed that these hybrid molecular materials hierarchically self-assembled into higher-order structures, in which, according to molecular dynamics simulations, disordered adhesive Mfp domains are exposed on the exterior of amyloid cores formed by CsgA. The assembled fibers displayed a strong adhesion energy $\left(\sim 20.9 \mathrm{~mJ} / \mathrm{m}^{2}\right)$, which was 1.5 times greater than existing bio-inspired and bio-derived protein-based underwater adhesives. Dr. Chao suggested that this study would inspire new design strategies for biomimetic materials.

One of major tasks in synthetic biology is to advance the technology for "writing DNA". While cloning has been a defining tool in molecular biology, the cloning of long DNA fragments, especially those contain large gene clusters that are of particular importance to synthetic and chemical biology efforts for engineering organisms, has been challenging. In the last talk of this section, Dr. Ting Zhu (Tsinghua University, China) presented a technique that allowed the targeted cloning of near-arbitrary, long bacterial genomic sequences $(50-150 \mathrm{~kb})$ to be accomplished in a single step [24]. This method utilized RNAguided Cas9 nucleas to cut target DNA from bacterial chromosomes, and then the excised DNA fragment was ligated to the cloning vector by Gibson assembly. Dr. Zhu suggested that this technique can be an effective molecular tool for the targeted cloning of large gene clusters that are expensive to synthesize by gene synthesis or difficult to obtain directly by traditional PCR and restriction-enzyme-based methods.

\section{LIGHTENING TALKS}

Four lightening talks were chosen from meeting abstracts. Mr. Weixi Liao (Tsinghua University, China) tried to use insulators, the non-coding gene regulatory elements in genome, to facilitate modular design of synthetic gene circuit by preventing unexpected interactions between different regulatory units. They functionally characterized 
the out-of-boundary regulatory interactions between different combinations of 6 promoters and 6 insulators, and showed that the interactions displayed a thresholdlike effect, and insulators influenced the threshold position. They also tested the function of insulators in both cascade and toggle-switch circuits. Mr. Liren Wang (East China Normal University, China) introduced their work to delete large genomic DNA fragment (up to $95 \mathrm{~kb}$ ) in mice to study the function of lncRNAs and formyl peptide receptors in vivo using optimized CRISPR/Cas system [25]. They also combined the Cre-Loxp system with a gene-trap strategy to provide a conditional knockout/reporter system suitable for mosaic mutation analysis. Mr. Zhi-Qiang Xiong (Shanghai Institutes for Biological Sciences, Chinese Academy of Sciences, China) adopted an omics approach based on in silico genome-scale metabolic analysis and comparative transcriptome to systematically investigate the problematic interactions between erythromycin precursor 6-deoxyerythronolide B (6dEB) module and native modules in E. coli. By modulating the identified targets, they successfully increase the yield of $6 \mathrm{dEB}$ compared to that of the original strain [26]. In the last lightening talk, Mr. Pei Du (Institute of Microbiology, Chinese Academy of Sciences, China) reported the design of programmable translational activator composed of a RNA endoribonuclease (Csy4) and two exchangeable RNA modules [27]. Among the three parts, Csy4, as a member of Cas6 endoribonuclease, cleaves a specific site within its recognition site. This cleavage will result in another cisrepressed RNA module (crRNA) releasing from the masked ribosome binding site (RBS), and the translation of the downstream gene will be activated. With modularized design and predictable functionality, this system can be integrated into the sophisticated genetic circuits.

\section{CONCLUSION}

The first SynBioYSF was a great success to brew and exchange ideas and knowledge among the synthetic biology community, especially young scholars in China. From 2015 forward, SynBioYSF will be hosted in different places by local organizers. As we wrote this summary, the second SynBioYSF has been planned to be held in Beijing, China, hosted by Peking University and Institute of Microbiology, Chinese Academy of Sciences. We believe that the second SynBioYSF will continue to attract attendees with diverse research backgrounds in the field of synthetic biology.

\section{ACKOWLEDGEMENTS}

The first SynBioYSF was supported by Tsinghua National Lab for Information Science and Technology, School of Life Sciences and
Department of Automation in Tsinghua University, THU-PKU Center for Life Sciences, Chinese Association for Artificial Intelligence, and Chinese Society of Biotechnology. We also thank Novozymes China, Beckman Coulter, Wuxi Qinglan Biotech, SyngenTech, Genewiz, Synbio Tech and Beijing Genomic Institute for their general sponsorships.

\section{REFERENCES}

1. Zhang, H., Lin, M., Shi, H., Ji, W., Huang, L., Zhang, X., Shen, S., Gao, R., Wu, S., Tian, C., et al. (2014) Programming a Pavlovian-like conditioning circuit in Escherichia coli. Nat. Commun., 5, 3102

2. Pardee, K., Green, A. A., Ferrante, T., Cameron, D. E., DaleyKeyser, A., Yin, P. and Collins, J. J. (2014) Paper-based synthetic gene networks. Cell, 159, 940-954

3. Sun, W., Boulais, E., Hakobyan, Y., Wang, W. L., Guan, A., Bathe, M. and Yin, P. (2014) Casting inorganic structures with DNA molds Science, 346, 1258361

4. Iinuma, R., Ke, Y., Jungmann, R., Schlichthaerle, T., Woehrstein, J. B. and Yin, P. (2014) Polyhedra self-assembled from DNA tripods and characterized with 3D DNA-PAINT. Science, 344, 65-69

5. Jungmann, R., Avendaño, M. S., Woehrstein, J. B., Dai, M., Shih, W. M. and Yin, P. (2014) Multiplexed 3D cellular super-resolution imaging with DNA-PAINT and Exchange-PAINT. Nat. Methods, 11, 313-318

6. Yang, Y., Ding, Y., Hu, Y., Cao, B., Rice, S. A., Kjelleberg, S. and Song, H. (2015b) Enhancing bidirectional electron transfer of Shewanella oneidensis by a synthetic flavin pathway. ACS Synth. Biol., 4, 815-823

7. Yong, Y. C., Yu, Y. Y., Zhang, X. and Song, H. (2014) Highly active bidirectional electron transfer by a self-assembled electroactive reduced-graphene-oxide-hybridized biofilm. Angew. Chem. Int. Ed. Engl., 53, 4480-4483

8. Guo, Z., Li, J., Qin, H., Wang, M., Lv, X., Li, X. and Chen, Y. (2015b) Biosynthesis of the carbamoylated D-gulosamine moiety of streptothricins: involvement of a guanidino-N-glycosyltransferase and an Nacetyl-D-gulosamine deacetylase. Angew. Chem. Int. Ed. Engl., 54, $5175-5178$

9. Yu, Y., Cui, C., Liu, X., Petrik, I. D., Wang, J. and Lu, Y. (2015) A designed metalloenzyme achieving the catalytic rate of a native enzyme. J. Am. Chem. Soc., 137, 11570-11573

10. Gilbert, L. A., Horlbeck, M. A., Adamson, B., Villalta, J. E., Chen, Y., Whitehead, E. H., Guimaraes, C., Panning, B., Ploegh, H. L., Bassik, M. C., et al. (2014) Genome-scale CRISPR-mediated control of gene repression and activation. Cell, 159, 647-661

11. Qi, L. S., Larson, M. H., Gilbert, L. A., Doudna, J. A., Weissman, J. S., Arkin, A. P. and Lim, W. A. (2013) Repurposing CRISPR as an RNAguided platform for sequence-specific control of gene expression. Cell, $152,1173-1183$

12. Zalatan, J. G., Lee, M. E., Almeida, R., Gilbert, L. A., Whitehead, E. H., La Russa, M., Tsai, J. C., Weissman, J. S., Dueber, J. E., Qi, L. S., et al. (2015) Engineering complex synthetic transcriptional programs with CRISPR RNA scaffolds. Cell, 160, 339-350

13. Ren, Q., Li, C., Yuan, P., Cai, C., Zhang, L., Luo, G. G. and Wei, W. (2015) A Dual-reporter system for real-time monitoring and highthroughput CRISPR/Cas9 library screening of the hepatitis C virus. Sci Rep, 5, 8865

14. Zhou, Y., Zhu, S., Cai, C., Yuan, P., Li, C., Huang, Y. and Wei, W. (2014) High-throughput screening of a CRISPR/Cas9 library for functional genomics in human cells. Nature, 509, 487-491

15. Yuan, Y., Liu, B., Xie, P., Zhang, M. Q., Li, Y., Xie, Z. and Wang, X. (2015) Model-guided quantitative analysis of microRNA-mediated 
regulation on competing endogenous RNAs using a synthetic gene circuit. Proc. Natl. Acad. Sci. USA, 112, 3158-3163

16. Chang, H. Y., Li, M. H., Huang, T. C., Hsu, C. L., Tsai, S. R., Lee, S. C., Huang, H. C. and Juan, H. F. (2015) Quantitative proteomics reveals middle infrared radiation-interfered networks in breast cancer cells. J. Proteome Res., 14, 1250-1262

17. Huang, C. T., Oyang, Y. J., Huang, H. C. and Juan, H. F. (2014) MicroRNA-mediated networks underlie immune response regulation in papillary thyroid carcinoma. Sci. Rep., 4, 6495

18. Lin, L. L., Hsia, C. R., Hsu, C. L., Huang, H. C. and Juan, H. F. (2015) Integrating transcriptomics and proteomics to show that tanshinone IIA suppresses cell growth by blocking glucose metabolism in gastric cancer cells. BMC Genomics, 16, 41

19. Yang, K. C., Hsu, C. L., Lin, C. C., Juan, H. F. and Huang, H. C. (2014) Mirin: identifying microRNA regulatory modules in protein-protein interaction networks. Bioinformatics, 30, 2527-2528

20. Ye, H., Charpin-El Hamri, G., Zwicky, K., Christen, M., Folcher, M. and Fussenegger, M. (2013) Pharmaceutically controlled designer circuit for the treatment of the metabolic syndrome. Proc. Natl. Acad. Sci. USA, 110, 141-146

21. Yao, Y., Jin, S., Long, H., Yu, Y., Zhang, Z., Cheng, G., Xu, C., Ding, Y., Guan, Q., Li, N., et al. (2015) RNAe: an effective method for targeted protein translation enhancement by artificial non-coding RNA with SINEB2 repeat. Nucleic Acids Res., 43, e58

22. Guo, Y., Dong, J., Zhou, T., Auxillos, J., Li, T., Zhang, W., Wang, L., Shen, Y., Luo, Y., Zheng, Y., et al. (2015a) YeastFab: the design and construction of standard biological parts for metabolic engineering in Saccharomyces cerevisiae. Nucleic Acids Res., 43, e88

23. Zhong, C., Gurry, T., Cheng, A. A., Downey, J., Deng, Z., Stultz, C. M. and $\mathrm{Lu}, \mathrm{T}$. K. (2014) Strong underwater adhesives made by selfassembling multi-protein nanofibres. Nat. Nanotechnol., 9, 858-866

24. Jiang, W., Zhao, X., Gabrieli, T., Lou, C., Ebenstein, Y. and Zhu, T. F. (2015) Cas9-Assisted targeting of chromosome segments CATCH enables one-step targeted cloning of large gene clusters. Nat. Commun., 6,8101

25. Shao, Y., Guan, Y., Wang, L., Qiu, Z., Liu, M., Chen, Y., Wu, L., Li, Y., Ma, X., Liu, M., et al. (2014) CRISPR/Cas-mediated genome editing in the rat via direct injection of one-cell embryos. Nat. Protoc., 9, 24932512

26. Yang, J., Xiong, Z. Q., Song, S. J., Wang, J. F., Lv, H. J. and Wang, Y. (2015a) Improving heterologous polyketide production in Escherichia coli by transporter engineering. Appl. Microbiol. Biotechnol., 99, 8691-8700

27. Du, P., Miao, C., Lou, Q., Wang, Z. and Lou, C. (2015) Engineering translational activators with CRISPR-Cas system. ACS Synth. Biol., doi: 10.1021 acssynbio.5b00130 